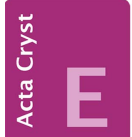
COMMUNICATIONS

ISSN 2056-9890

\section{Crystal structure of 3-[2-(thiophen-3-yl)- ethynyl]-2H-chromen-2-one}

\author{
Ignez Caracelli, ${ }^{a}$ Stella H. Maganhi, ${ }^{a}$ Hélio A. Stefani, ${ }^{b}$ \\ Karina Gueogjian ${ }^{\mathrm{b}}$ and Edward R. T. Tiekink ${ }^{\mathrm{C}}$

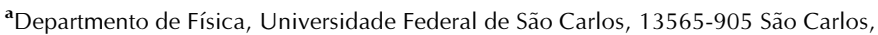 \\ SP, Brazil, 'b Departamento de Farmácia, Faculdade de Ciências Farmacêuticas, \\ Universidade de São Paulo, 05508-900 São Paulo, SP, Brazil, and ${ }^{\mathbf{c}}$ Department of \\ Chemistry, University of Malaya, 50603 Kuala Lumpur, Malaysia. *Correspondence \\ e-mail: ignez@ufscar.br
}

Received 26 January 2015; accepted 2 February 2015

Edited by H. Stoeckli-Evans, University of Neuchâtel, Switzerland

In the title compound, $\mathrm{C}_{15} \mathrm{H}_{8} \mathrm{O}_{2} \mathrm{~S}$, the coumarin moiety is approximately planar (r.m.s. deviation of the 11 non- $\mathrm{H}$ atoms $=$ $0.025 \AA$ ) and is slightly inclined with respect to the plane of the thiophen-3-yl ring, forming a dihedral angle of $11.75(8)^{\circ}$. In the crystal, the three-dimensional architecture features a combination of coumarin-thiophene $\mathrm{C}-\mathrm{H} \cdots \pi$ and $\pi-\pi$ [inter-centroid distance $=3.6612(12) \AA]$ interactions.

Keywords: crystal structure; coumarin; asymmetric alkyne; $\mathrm{C}-\mathrm{H} \cdots \pi$ interactions; $\pi-\pi$ interactions.

CCDC reference: 1046686

\section{Related literature}

For the wide range of different biological activities of coumarins, see: Wu et al. (2009); Roussaki et al. (2014). For background to our ongoing interest in the synthesis and crystal structures of coumarin derivatives, see: Stefani et al. (2012); Caracelli et al. (2015). For the synthesis of the title compound, see: Gueogiian (2011).

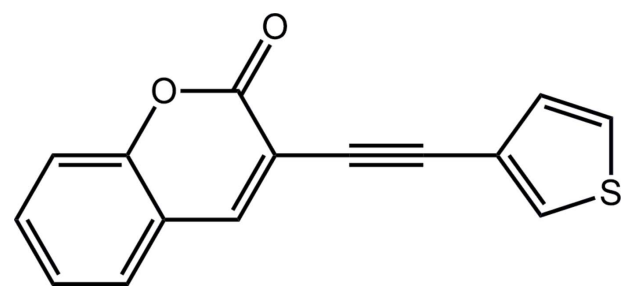

\section{Experimental}

2.1. Crystal data

$\mathrm{C}_{15} \mathrm{H}_{8} \mathrm{O}_{2} \mathrm{~S}$

$M_{r}=252.27$

Monoclinic, $P 2_{1} / c$

$a=10.7726(6) \AA$

$b=9.7572(3) \AA$

$c=12.2084(5) \AA$

$\beta=115.547(6)^{\circ}$

$$
\begin{aligned}
& V=1157.77(11) \AA^{3} \\
& Z=4 \\
& \text { Cu K } \alpha \text { radiation } \\
& \mu=2.40 \mathrm{~mm}^{-1} \\
& T=100 \mathrm{~K} \\
& 0.25 \times 0.15 \times 0.05 \mathrm{~mm}
\end{aligned}
$$

\subsection{Data collection}

Agilent CCD diffractometer Absorption correction: multi-scan (CrysAlis PRO; Agilent, 2011)

$T_{\min }=0.338, T_{\max }=1.000$

4511 measured reflections 2373 independent reflections 2108 reflections with $I>2 \sigma(I)$ $R_{\text {int }}=0.023$

\subsection{Refinement}

$R\left[F^{2}>2 \sigma\left(F^{2}\right)\right]=0.050$

$w R\left(F^{2}\right)=0.156$

$S=1.06$

2373 reflections

163 parameters

$\mathrm{H}$-atom parameters constrained

$\Delta \rho_{\max }=0.42 \mathrm{e} \AA^{-3}$

$\Delta \rho_{\min }=-0.57$ e $\AA^{-3}$

Table 1

Hydrogen-bond geometry $\left(\AA,{ }^{\circ}\right)$.

$C g 1$ is the centroid of ring S1,C1 $\cdots \mathrm{C} 4$.

\begin{tabular}{lllll}
\hline$D-\mathrm{H} \cdots A$ & $D-\mathrm{H}$ & $\mathrm{H} \cdots A$ & $D \cdots A$ & $D-\mathrm{H} \cdots A$ \\
\hline $\mathrm{C} 14-\mathrm{H} 14 \cdots C g 1^{\mathrm{i}}$ & 0.95 & 2.89 & $3.701(2)$ & 144 \\
\hline
\end{tabular}

Symmetry code: (i) $x-1,-y+\frac{1}{2}, z-\frac{1}{2}$.

Data collection: CrysAlis PRO (Agilent, 2011); cell refinement: CrysAlis PRO; data reduction: CrysAlis PRO; program(s) used to solve structure: SIR2014 (Burla et al., 2015); program(s) used to refine structure: SHELXL2014 (Sheldrick, 2015); molecular graphics: ORTEP-3 for Windows (Farrugia, 2012) and DIAMOND (Brandenburg, 2006); software used to prepare material for publication: MarvinSketch (ChemAxon, 2010) and publCIF (Westrip, 2010).

\title{
Acknowledgements
}

The Brazilian agencies CNPq (306121/2013-2 to IC and 308320/2010-7 to HAS), FAPESP (2012/00424-2) and CAPES are acknowledged for financial support.

Supporting information for this paper is available from the IUCr electronic archives (Reference: SU5073).

\section{References}

Agilent (2011). CrysAlis PRO. Agilent Technologies, Yarnton, England. Brandenburg, K. (2006). DIAMOND. Crystal Impact GbR, Bonn, Germany.

Burla, M. C., Caliandro, R., Carrozzini, B., Cascarano, G. L., Cuocci, C., Giacovazzo, C., Mallamo, M., Mazzone, A. \& Polidori, G. (2015). J. Appl. Cryst. 48, 306-309.

Caracelli, I., Maganhi, S. H., Stefani, H. A., Gueogjian, K. \& Tiekink, E. R. T. (2015). Acta Cryst. E71, o90-091.

ChemAxon (2010). Marvinsketch. http://www.chemaxon.com.

Farrugia, L. J. (2012). J. Appl. Cryst. 45, 849-854.

Gueogjian, K. (2011). PhD thesis, University of São Paulo, Brazil. 
Roussaki, M., Zelianaios, K., Kavetsou, E., Hamilakis, S., Hadjipavlou-Litina, D., Kontogiorgis, C., Liargkova, T. \& Detsi, A. (2014). Bioorg. Med. Chem. 22, 6586-6594.

Sheldrick, G. M. (2015). Acta Cryst. C71, 3-8.

Stefani, H. A., Gueogjan, K., Manarin, F., Farsky, S. H. P., ZukermanSchpector, J., Caracelli, I., Pizano Rodrigues, S. R., Muscará, M. N., Teixeira,
S. A., Santin, J. R., Machado, I. D., Bolonheis, S. M., Curi, R. \& Vinolo, M. A. (2012). Eur. J. Med. Chem. 58, 117-127.

Westrip, S. P. (2010). J. Appl. Cryst. 43, 920-925.

Wu, L., Wang, X., Xu, W., Farzaneh, F. \& Xu, R. (2009). Curr. Med. Chem. 16, 4236-4260. 


\section{supporting information}

Acta Cryst. (2015). E71, o154-o155 [doi:10.1107/S2056989015002157]

\section{Crystal structure of 3-[2-(thiophen-3-yl)ethynyl]-2H-chromen-2-one}

\section{Ignez Caracelli, Stella H. Maganhi, Hélio A. Stefani, Karina Gueogjian and Edward R. T. Tiekink}

\section{S1. Synthesis and crystallization}

The title compound was prepared as per Gueogjian (2011). 3-Bromo coumarin (112.5 mg, $0.5 \mathrm{mmol}$ ), potassium trifluoroborate salt $(0.55 \mathrm{mmol}), \mathrm{PdCl}_{2}$ (dppf). $\mathrm{CH}_{2} \mathrm{Cl}_{2}(41 \mathrm{mg}, 10 \mathrm{~mol} \%), i-\operatorname{Pr} 2 \mathrm{NEt}(0.3 \mathrm{~mL}, 1.5 \mathrm{mmol})$ and 1,4-dioxane $/ \mathrm{H}_{2} \mathrm{O}$ $(2 / 1,3 \mathrm{~mL})$, in acetonitrile $(20 \mathrm{~mL})$ were added to a two-necked round-bottomed flask equipped with a reflux condenser under $\mathrm{N}_{2}$. The reaction mixture was heated under reflux at $353 \mathrm{~K}$, and was monitored by TLC and GC analysis. After the consumption of the 3-bromocoumarin, the mixture was extracted twice with ethyl acetate $(50 \mathrm{~mL})$. The organic phase was separated, dried over $\mathrm{MgSO}_{4}$ and concentrated under vacuum. The residue was purified by flash chromatography (ethyl acetate/hexane 10:90). The title compound was obtained as a dark-yellow solid in 53\% yield. Suitable crystals were obtained by slow evaporation from a mixture of ethyl acetate/hexane.

\section{S2. Refinement}

C-bound $\mathrm{H}$-atoms were placed in calculated positions $(\mathrm{C}-\mathrm{H}=0.95 \AA)$ and were included in the refinement in the riding model approximation, with $U_{i s o}(\mathrm{H})=1.2 U_{e q}(\mathrm{C})$.

\section{S3. Comment}

Coumarins are heterocycles presenting a wide range of different biological activities (Wu et al., 2009; Roussaki et al., 2014). As part of our on-going interest in the synthesis and crystal structures of coumarin derivatives with biological activity (Stefani et al., 2012; Caracelli et al., 2015) the title compound was synthesized (Gueogjian, 2011).

\section{S4. Experimental}

The title compound was prepared as per Gueogjian (2011). 3-Bromo coumarin (112.5 mg, $0.5 \mathrm{mmol}$ ), potassium trifluoroborate salt $(0.55 \mathrm{mmol}), \mathrm{PdCl}_{2}$ (dppf) $\cdot \mathrm{CH}_{2} \mathrm{Cl}_{2}(41 \mathrm{mg}, 10 \mathrm{~mol} \%), i-\operatorname{Pr} 2 \mathrm{NEt}(0.3 \mathrm{ml}, 1.5 \mathrm{mmol})$ and 1,4-dioxane $/ \mathrm{H}_{2} \mathrm{O}$ $(2 / 1,3 \mathrm{ml})$, in acetonitrile $(20 \mathrm{ml})$ were added to a two-necked round-bottomed flask equipped with a reflux condenser under $\mathrm{N}_{2}$. The reaction mixture was heated under reflux at $353 \mathrm{~K}$, and was monitored by TLC and GC analysis. After the consumption of the 3-bromocoumarin, the mixture was extracted twice with ethyl acetate $(50 \mathrm{ml})$. The organic phase was separated, dried over $\mathrm{MgSO}_{4}$ and concentrated under vacuum. The residue was purified by flash chromatography (ethyl acetate/hexane 10:90). The title compound was obtained as a dark-yellow solid in 53\% yield. Suitable crystals were obtained by slow evaporation from a mixture of ethyl acetate/hexane. 


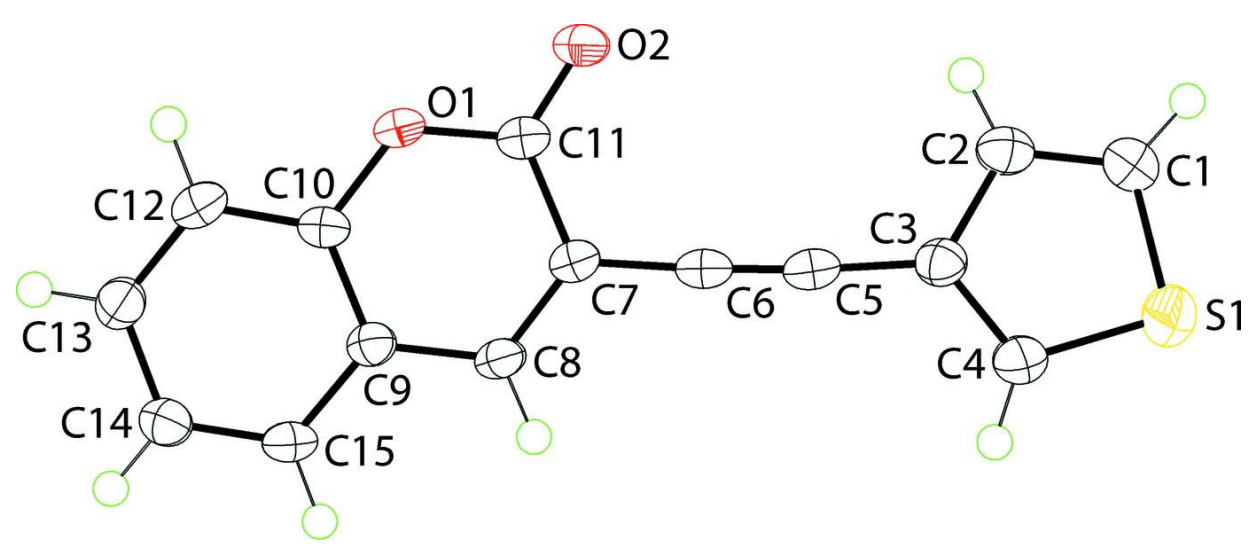

\section{Figure 1}

Molecular structure of the title compound showing atom labelling and displacement ellipsoids at the $70 \%$ probability level.

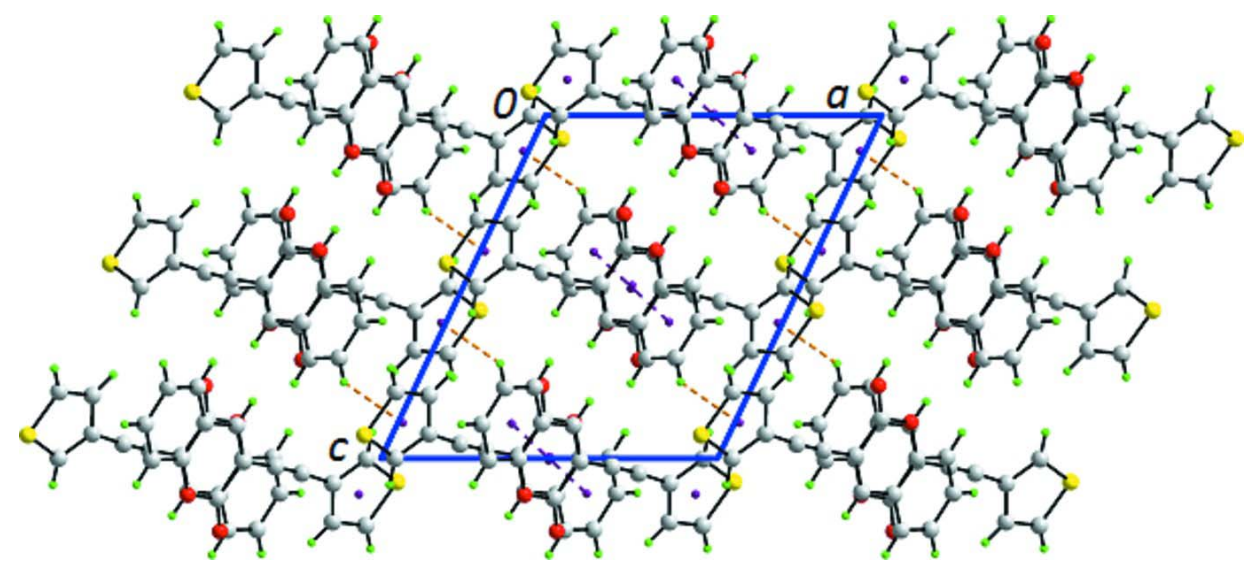

\section{Figure 2}

A view in projection down the $b$ axis of the unit-cell contents. The $\pi-\pi$ and $\mathrm{C}-\mathrm{H} \cdots \pi$ interactions are shown as purple and orange dashed lines, respectively.

\section{3-[2-(Thiophen-3-yl)ethynyl]-2H-chromen-2-one}

Crystal data

$\mathrm{C}_{15} \mathrm{H}_{8} \mathrm{O}_{2} \mathrm{~S}$

$M_{r}=252.27$

Monoclinic, $P 2_{1} / c$

$a=10.7726(6) \AA$

$b=9.7572(3) \AA$

$c=12.2084(5) \AA$

$\beta=115.547(6)^{\circ}$

$V=1157.77(11) \AA^{3}$

$Z=4$
$F(000)=520$

$D_{\mathrm{x}}=1.447 \mathrm{Mg} \mathrm{m}^{-3}$

$\mathrm{Cu} K \alpha$ radiation, $\lambda=1.54184 \AA$

Cell parameters from 2362 reflections

$\theta=4.0-76.0^{\circ}$

$\mu=2.40 \mathrm{~mm}^{-1}$

$T=100 \mathrm{~K}$

Prism, dark yellow

$0.25 \times 0.15 \times 0.05 \mathrm{~mm}$ 


\section{Data collection \\ Agilent CCD \\ diffractometer \\ Radiation source: SuperNova $(\mathrm{Cu}) \mathrm{X}$-ray \\ Source \\ $\omega$ scans \\ Absorption correction: multi-scan \\ (CrysAlis PRO; Agilent, 2011) \\ $T_{\min }=0.338, T_{\max }=1.000$}

\section{Refinement}

Refinement on $F^{2}$

Least-squares matrix: full

$R\left[F^{2}>2 \sigma\left(F^{2}\right)\right]=0.050$

$w R\left(F^{2}\right)=0.156$

$S=1.06$

2373 reflections

163 parameters

0 restraints
4511 measured reflections

2373 independent reflections

2108 reflections with $I>2 \sigma(I)$

$R_{\text {int }}=0.023$

$\theta_{\max }=76.2^{\circ}, \theta_{\min }=4.6^{\circ}$

$h=-13 \rightarrow 11$

$k=-12 \rightarrow 10$

$l=-15 \rightarrow 14$

Primary atom site location: structure-invariant direct methods

Hydrogen site location: inferred from neighbouring sites

$\mathrm{H}$-atom parameters constrained

$w=1 /\left[\sigma^{2}\left(F_{\mathrm{o}}^{2}\right)+(0.1031 P)^{2}+0.5663 P\right]$

where $P=\left(F_{\mathrm{o}}^{2}+2 F_{\mathrm{c}}^{2}\right) / 3$

$(\Delta / \sigma)_{\max }<0.001$

$\Delta \rho_{\max }=0.42 \mathrm{e} \AA^{-3}$

$\Delta \rho_{\min }=-0.57$ e $\AA^{-3}$

Special details

Geometry. All e.s.d.'s (except the e.s.d. in the dihedral angle between two 1.s. planes) are estimated using the full covariance matrix. The cell e.s.d.'s are taken into account individually in the estimation of e.s.d.'s in distances, angles and torsion angles; correlations between e.s.d.'s in cell parameters are only used when they are defined by crystal symmetry. An approximate (isotropic) treatment of cell e.s.d.'s is used for estimating e.s.d.'s involving l.s. planes.

Fractional atomic coordinates and isotropic or equivalent isotropic displacement parameters $\left(\AA^{2}\right)$

\begin{tabular}{|c|c|c|c|c|}
\hline & $x$ & $y$ & $z$ & $U_{\text {iso }} * / U_{\text {eq }}$ \\
\hline $\mathrm{S} 1$ & $1.08150(6)$ & $0.79946(6)$ & $0.56811(5)$ & $0.0338(2)$ \\
\hline $\mathrm{O} 1$ & $0.47823(14)$ & $0.23152(14)$ & $0.60599(12)$ & $0.0194(3)$ \\
\hline $\mathrm{O} 2$ & $0.62583(14)$ & $0.39103(15)$ & $0.71228(12)$ & $0.0236(3)$ \\
\hline $\mathrm{C} 1$ & $1.0505(2)$ & 0.80962 (19) & 0.69506 (19) & $0.0225(4)$ \\
\hline H1 & 1.0976 & 0.8684 & 0.7624 & $0.027 *$ \\
\hline $\mathrm{C} 2$ & $0.9466(2)$ & $0.7191(2)$ & 0.68413 (19) & $0.0236(4)$ \\
\hline $\mathrm{H} 2$ & 0.9143 & 0.7081 & 0.7448 & $0.028^{*}$ \\
\hline C3 & 0.8928 (2) & $0.64361(19)$ & $0.57262(18)$ & $0.0204(4)$ \\
\hline $\mathrm{C} 4$ & $0.9581(2)$ & $0.6785(2)$ & 0.50099 (19) & $0.0274(5)$ \\
\hline $\mathrm{H} 4$ & 0.9368 & 0.6396 & 0.4236 & $0.033 *$ \\
\hline $\mathrm{C} 5$ & $0.7859(2)$ & $0.5442(2)$ & $0.54250(17)$ & $0.0207(4)$ \\
\hline C6 & $0.69803(19)$ & $0.4614(2)$ & $0.52352(16)$ & $0.0198(4)$ \\
\hline $\mathrm{C} 11$ & $0.57073(19)$ & $0.3326(2)$ & $0.61608(17)$ & $0.0186(4)$ \\
\hline $\mathrm{C} 7$ & $0.59579(19)$ & 0.35987 (19) & $0.50841(17)$ & $0.0183(4)$ \\
\hline $\mathrm{C} 8$ & $0.5239(2)$ & $0.29061(19)$ & $0.40315(18)$ & $0.0194(4)$ \\
\hline H8 & 0.5380 & 0.3115 & 0.3334 & $0.023 *$ \\
\hline C9 & $0.4272(2)$ & 0.18649 (19) & $0.39642(18)$ & $0.0183(4)$ \\
\hline $\mathrm{C} 15$ & $0.3539(2)$ & $0.1072(2)$ & $0.29190(17)$ & $0.0211(4)$ \\
\hline H15 & 0.3642 & 0.1249 & 0.2198 & $0.025 *$ \\
\hline $\mathrm{C} 14$ & $0.2672(2)$ & 0.00402 (19) & $0.29346(18)$ & $0.0216(4)$ \\
\hline
\end{tabular}


supporting information

\begin{tabular}{lllll}
$\mathrm{H} 14$ & 0.2187 & -0.0497 & 0.2229 & $0.026^{*}$ \\
$\mathrm{C} 13$ & $0.2510(2)$ & $-0.0214(2)$ & $0.39989(18)$ & $0.0224(4)$ \\
$\mathrm{H} 13$ & 0.1905 & -0.0920 & 0.4004 & $0.027^{*}$ \\
$\mathrm{C} 12$ & $0.3219(2)$ & $0.0550(2)$ & $0.50393(18)$ & $0.0218(4)$ \\
$\mathrm{H} 12$ & 0.3114 & 0.0371 & 0.5759 & $0.026^{*}$ \\
$\mathrm{C} 10$ & $0.40856(19)$ & $0.1581(2)$ & $0.50072(17)$ & $0.0185(4)$ \\
\hline
\end{tabular}

Atomic displacement parameters $\left(\AA^{2}\right)$

\begin{tabular}{lllllll}
\hline & $U^{11}$ & $U^{22}$ & $U^{33}$ & $U^{12}$ & $U^{13}$ & $U^{23}$ \\
\hline S1 & $0.0356(4)$ & $0.0357(4)$ & $0.0303(4)$ & $-0.0118(2)$ & $0.0144(3)$ & $0.0005(2)$ \\
O1 & $0.0231(7)$ & $0.0234(7)$ & $0.0148(6)$ & $-0.0009(5)$ & $0.0109(5)$ & $0.0002(5)$ \\
O2 & $0.0262(7)$ & $0.0299(8)$ & $0.0159(7)$ & $-0.0019(6)$ & $0.0102(6)$ & $-0.0023(6)$ \\
C1 & $0.0212(9)$ & $0.0219(9)$ & $0.0222(10)$ & $0.0033(7)$ & $0.0072(8)$ & $-0.0011(7)$ \\
C2 & $0.0254(10)$ & $0.0257(9)$ & $0.0209(10)$ & $0.0022(8)$ & $0.0112(8)$ & $-0.0019(7)$ \\
C3 & $0.0221(9)$ & $0.0205(9)$ & $0.0182(9)$ & $0.0010(7)$ & $0.0082(7)$ & $0.0021(7)$ \\
C4 & $0.0334(11)$ & $0.0304(10)$ & $0.0194(10)$ & $-0.0080(9)$ & $0.0122(9)$ & $-0.0008(8)$ \\
C5 & $0.0244(9)$ & $0.0241(9)$ & $0.0154(8)$ & $0.0034(8)$ & $0.0104(7)$ & $0.0018(7)$ \\
C6 & $0.0246(10)$ & $0.0218(9)$ & $0.0142(8)$ & $0.0040(7)$ & $0.0096(7)$ & $0.0005(7)$ \\
C11 & $0.0211(9)$ & $0.0202(9)$ & $0.0159(9)$ & $0.0030(7)$ & $0.0092(7)$ & $0.0009(7)$ \\
C7 & $0.0208(9)$ & $0.0197(9)$ & $0.0164(9)$ & $0.0016(7)$ & $0.0099(7)$ & $0.0016(7)$ \\
C8 & $0.0228(9)$ & $0.0226(9)$ & $0.0160(9)$ & $0.0000(7)$ & $0.0113(8)$ & $0.0006(7)$ \\
C9 & $0.0207(9)$ & $0.0183(8)$ & $0.0174(9)$ & $0.0011(7)$ & $0.0098(7)$ & $0.0002(7)$ \\
C15 & $0.0245(9)$ & $0.0255(9)$ & $0.0146(8)$ & $0.0006(7)$ & $0.0097(7)$ & $-0.0003(7)$ \\
C14 & $0.0238(9)$ & $0.0213(9)$ & $0.0194(9)$ & $0.0002(7)$ & $0.0092(7)$ & $-0.0020(7)$ \\
C13 & $0.0227(9)$ & $0.0230(9)$ & $0.0224(10)$ & $-0.0020(7)$ & $0.0105(8)$ & $0.0014(7)$ \\
C12 & $0.0255(10)$ & $0.0235(9)$ & $0.0198(9)$ & $0.0026(8)$ & $0.0130(8)$ & $0.0048(7)$ \\
C10 & $0.0210(9)$ & $0.0200(9)$ & $0.0148(9)$ & $0.0019(7)$ & $0.0081(7)$ & $-0.0008(7)$ \\
& & & & & & \\
\hline
\end{tabular}

Geometric parameters $\left(\AA,{ }^{\circ}\right)$

\begin{tabular}{llll}
\hline $\mathrm{S} 1-\mathrm{C} 4$ & $1.701(2)$ & $\mathrm{C} 11-\mathrm{C} 7$ & $1.476(3)$ \\
$\mathrm{S} 1-\mathrm{C} 1$ & $1.723(2)$ & $\mathrm{C} 7-\mathrm{C} 8$ & $1.360(3)$ \\
$\mathrm{O} 1-\mathrm{C} 11$ & $1.369(2)$ & $\mathrm{C} 8-\mathrm{C} 9$ & $1.432(3)$ \\
$\mathrm{O} 1-\mathrm{C} 10$ & $1.377(2)$ & $\mathrm{C} 8-\mathrm{H} 8$ & 0.9500 \\
$\mathrm{O} 2-\mathrm{C} 11$ & $1.206(2)$ & $\mathrm{C} 9-\mathrm{C} 10$ & $1.400(3)$ \\
$\mathrm{C} 1-\mathrm{C} 2$ & $1.387(3)$ & $\mathrm{C} 9-\mathrm{C} 15$ & $1.408(3)$ \\
$\mathrm{C} 1-\mathrm{H} 1$ & 0.9500 & $\mathrm{C} 15-\mathrm{C} 14$ & $1.378(3)$ \\
$\mathrm{C} 2-\mathrm{C} 3$ & $1.432(3)$ & $\mathrm{C} 15-\mathrm{H} 15$ & 0.9500 \\
$\mathrm{C} 2-\mathrm{H} 2$ & 0.9500 & $\mathrm{C} 14-\mathrm{C} 13$ & $1.406(3)$ \\
$\mathrm{C} 3-\mathrm{C} 4$ & $1.381(3)$ & $\mathrm{C} 14-\mathrm{H} 14$ & 0.9500 \\
$\mathrm{C} 3-\mathrm{C} 5$ & $1.426(3)$ & $\mathrm{C} 13-\mathrm{C} 12$ & $1.384(3)$ \\
$\mathrm{C} 4-\mathrm{H} 4$ & 0.9500 & $\mathrm{C} 13-\mathrm{H} 13$ & 0.9500 \\
$\mathrm{C} 5-\mathrm{C} 6$ & $1.189(3)$ & $\mathrm{C} 12-\mathrm{C} 10$ & $1.384(3)$ \\
$\mathrm{C} 6-\mathrm{C} 7$ & $1.433(3)$ & $\mathrm{C} 12-\mathrm{H} 12$ & 0.9500 \\
& & & $120.69(18)$ \\
$\mathrm{C} 4-\mathrm{S} 1-\mathrm{C} 1$ & $93.39(10)$ & $\mathrm{C} 7-\mathrm{C} 8-\mathrm{C} 9$ & 119.7 \\
$\mathrm{C} 11-\mathrm{O} 1-\mathrm{C} 10$ & $122.76(15)$ & $\mathrm{C} 7-\mathrm{C} 8-\mathrm{H} 8$ &
\end{tabular}




\begin{tabular}{|c|c|c|c|}
\hline $\mathrm{C} 2-\mathrm{C} 1-\mathrm{S} 1$ & $109.76(15)$ & $\mathrm{C} 9-\mathrm{C} 8-\mathrm{H} 8$ & 119.7 \\
\hline $\mathrm{C} 2-\mathrm{C} 1-\mathrm{H} 1$ & 125.1 & $\mathrm{C} 10-\mathrm{C} 9-\mathrm{C} 15$ & $118.13(18)$ \\
\hline $\mathrm{S} 1-\mathrm{C} 1-\mathrm{H} 1$ & 125.1 & $\mathrm{C} 10-\mathrm{C} 9-\mathrm{C} 8$ & $118.32(18)$ \\
\hline $\mathrm{C} 1-\mathrm{C} 2-\mathrm{C} 3$ & $113.49(19)$ & $\mathrm{C} 15-\mathrm{C} 9-\mathrm{C} 8$ & $123.50(18)$ \\
\hline $\mathrm{C} 1-\mathrm{C} 2-\mathrm{H} 2$ & 123.3 & $\mathrm{C} 14-\mathrm{C} 15-\mathrm{C} 9$ & $120.50(17)$ \\
\hline $\mathrm{C} 3-\mathrm{C} 2-\mathrm{H} 2$ & 123.3 & $\mathrm{C} 14-\mathrm{C} 15-\mathrm{H} 15$ & 119.8 \\
\hline $\mathrm{C} 4-\mathrm{C} 3-\mathrm{C} 5$ & $125.39(18)$ & $\mathrm{C} 9-\mathrm{C} 15-\mathrm{H} 15$ & 119.8 \\
\hline $\mathrm{C} 4-\mathrm{C} 3-\mathrm{C} 2$ & $111.53(18)$ & $\mathrm{C} 15-\mathrm{C} 14-\mathrm{C} 13$ & $119.78(18)$ \\
\hline $\mathrm{C} 5-\mathrm{C} 3-\mathrm{C} 2$ & $123.08(18)$ & $\mathrm{C} 15-\mathrm{C} 14-\mathrm{H} 14$ & 120.1 \\
\hline $\mathrm{C} 3-\mathrm{C} 4-\mathrm{S} 1$ & $111.83(16)$ & $\mathrm{C} 13-\mathrm{C} 14-\mathrm{H} 14$ & 120.1 \\
\hline $\mathrm{C} 3-\mathrm{C} 4-\mathrm{H} 4$ & 124.1 & $\mathrm{C} 12-\mathrm{C} 13-\mathrm{C} 14$ & $120.90(18)$ \\
\hline $\mathrm{S} 1-\mathrm{C} 4-\mathrm{H} 4$ & 124.1 & $\mathrm{C} 12-\mathrm{C} 13-\mathrm{H} 13$ & 119.6 \\
\hline $\mathrm{C} 6-\mathrm{C} 5-\mathrm{C} 3$ & $176.60(19)$ & $\mathrm{C} 14-\mathrm{C} 13-\mathrm{H} 13$ & 119.6 \\
\hline $\mathrm{C} 5-\mathrm{C} 6-\mathrm{C} 7$ & $176.52(19)$ & $\mathrm{C} 13-\mathrm{C} 12-\mathrm{C} 10$ & $118.53(17)$ \\
\hline $\mathrm{O} 2-\mathrm{C} 11-\mathrm{O} 1$ & $117.48(17)$ & $\mathrm{C} 13-\mathrm{C} 12-\mathrm{H} 12$ & 120.7 \\
\hline $\mathrm{O} 2-\mathrm{C} 11-\mathrm{C} 7$ & $125.43(18)$ & $\mathrm{C} 10-\mathrm{C} 12-\mathrm{H} 12$ & 120.7 \\
\hline $\mathrm{O} 1-\mathrm{C} 11-\mathrm{C} 7$ & $117.09(16)$ & $\mathrm{O} 1-\mathrm{C} 10-\mathrm{C} 12$ & $117.02(16)$ \\
\hline $\mathrm{C} 8-\mathrm{C} 7-\mathrm{C} 6$ & $123.92(17)$ & $\mathrm{O} 1-\mathrm{C} 10-\mathrm{C} 9$ & $120.80(17)$ \\
\hline $\mathrm{C} 8-\mathrm{C} 7-\mathrm{C} 11$ & $120.25(17)$ & $\mathrm{C} 12-\mathrm{C} 10-\mathrm{C} 9$ & $122.17(18)$ \\
\hline $\mathrm{C} 6-\mathrm{C} 7-\mathrm{C} 11$ & $115.83(16)$ & & \\
\hline $\mathrm{C} 4-\mathrm{S} 1-\mathrm{C} 1-\mathrm{C} 2$ & $0.47(17)$ & $\mathrm{C} 7-\mathrm{C} 8-\mathrm{C} 9-\mathrm{C} 10$ & $0.1(3)$ \\
\hline $\mathrm{S} 1-\mathrm{C} 1-\mathrm{C} 2-\mathrm{C} 3$ & $-0.5(2)$ & $\mathrm{C} 7-\mathrm{C} 8-\mathrm{C} 9-\mathrm{C} 15$ & $-177.24(18)$ \\
\hline $\mathrm{C} 1-\mathrm{C} 2-\mathrm{C} 3-\mathrm{C} 4$ & $0.3(3)$ & $\mathrm{C} 10-\mathrm{C} 9-\mathrm{C} 15-\mathrm{C} 14$ & $-0.5(3)$ \\
\hline $\mathrm{C} 1-\mathrm{C} 2-\mathrm{C} 3-\mathrm{C} 5$ & $179.59(18)$ & $\mathrm{C} 8-\mathrm{C} 9-\mathrm{C} 15-\mathrm{C} 14$ & $176.84(18)$ \\
\hline $\mathrm{C} 5-\mathrm{C} 3-\mathrm{C} 4-\mathrm{S} 1$ & $-179.21(16)$ & $\mathrm{C} 9-\mathrm{C} 15-\mathrm{C} 14-\mathrm{C} 13$ & $0.6(3)$ \\
\hline $\mathrm{C} 2-\mathrm{C} 3-\mathrm{C} 4-\mathrm{S} 1$ & $0.1(2)$ & $\mathrm{C} 15-\mathrm{C} 14-\mathrm{C} 13-\mathrm{C} 12$ & $-0.6(3)$ \\
\hline $\mathrm{C} 1-\mathrm{S} 1-\mathrm{C} 4-\mathrm{C} 3$ & $-0.30(18)$ & $\mathrm{C} 14-\mathrm{C} 13-\mathrm{C} 12-\mathrm{C} 10$ & $0.5(3)$ \\
\hline $\mathrm{C} 10-\mathrm{O} 1-\mathrm{C} 11-\mathrm{O} 2$ & $179.74(16)$ & $\mathrm{C} 11-\mathrm{O} 1-\mathrm{C} 10-\mathrm{C} 12$ & $177.52(16)$ \\
\hline $\mathrm{C} 10-\mathrm{O} 1-\mathrm{C} 11-\mathrm{C} 7$ & $-0.9(3)$ & $\mathrm{C} 11-\mathrm{O} 1-\mathrm{C} 10-\mathrm{C} 9$ & $-1.6(3)$ \\
\hline $\mathrm{O} 2-\mathrm{C} 11-\mathrm{C} 7-\mathrm{C} 8$ & $-177.71(19)$ & $\mathrm{C} 13-\mathrm{C} 12-\mathrm{C} 10-\mathrm{O} 1$ & $-179.57(16)$ \\
\hline $\mathrm{O} 1-\mathrm{C} 11-\mathrm{C} 7-\mathrm{C} 8$ & $3.0(3)$ & $\mathrm{C} 13-\mathrm{C} 12-\mathrm{C} 10-\mathrm{C} 9$ & $-0.5(3)$ \\
\hline $\mathrm{O} 2-\mathrm{C} 11-\mathrm{C} 7-\mathrm{C} 6$ & $2.3(3)$ & $\mathrm{C} 15-\mathrm{C} 9-\mathrm{C} 10-\mathrm{O} 1$ & $179.50(16)$ \\
\hline $\mathrm{O} 1-\mathrm{C} 11-\mathrm{C} 7-\mathrm{C} 6$ & $-177.02(15)$ & $\mathrm{C} 8-\mathrm{C} 9-\mathrm{C} 10-\mathrm{O} 1$ & $2.0(3)$ \\
\hline $\mathrm{C} 6-\mathrm{C} 7-\mathrm{C} 8-\mathrm{C} 9$ & $177.45(17)$ & $\mathrm{C} 15-\mathrm{C} 9-\mathrm{C} 10-\mathrm{C} 12$ & $0.5(3)$ \\
\hline $\mathrm{C} 11-\mathrm{C} 7-\mathrm{C} 8-\mathrm{C} 9$ & $-2.6(3)$ & $\mathrm{C} 8-\mathrm{C} 9-\mathrm{C} 10-\mathrm{C} 12$ & $-177.01(17)$ \\
\hline
\end{tabular}

Hydrogen-bond geometry $\left(A,{ }^{\circ}\right)$

$\mathrm{Cg} 1$ is the centroid of ring $\mathrm{S} 1, \mathrm{C} 1 \cdots \mathrm{C} 4$.

\begin{tabular}{lllll}
\hline$D-\mathrm{H} \cdots A$ & $D-\mathrm{H}$ & $\mathrm{H} \cdots A$ & $D \cdots A$ & $D-\mathrm{H} \cdots A$ \\
\hline $\mathrm{C} 14-\mathrm{H} 14 \cdots C g 1^{\mathrm{i}}$ & 0.95 & 2.89 & $3.701(2)$ & 144 \\
\hline
\end{tabular}

Symmetry code: (i) $x-1,-y+1 / 2, z-1 / 2$. 